\title{
High velocity impact of a composite sandwich panel
}

\author{
M. S. Hoo Fatt \& D. Sirivolu \\ Department of Mechanical Engineering, \\ The University of Akron, Ohio, USA
}

\begin{abstract}
Analytical solutions for the deformation response of a composite sandwich panel subjected to high velocity impact by a rigid blunt, cylindrical projectile are presented. The solution is derived from a two degrees-of-freedom model for the sandwich panel involving local indentation, core crushing and global bending/shear deformations. An example is given for a composite sandwich panel consisting of orthotropic E-glass vinyl ester facesheets and PVC H100 foam core and subjected to the high velocity impact of a blunt cylindrical projectile. The analytical solution for the local indentation and global deflection under the projectile was found to be within $15 \%$ of FEA results.
\end{abstract}

Keywords: high velocity impact, composite sandwich panels, analytical model.

\section{Introduction}

Light weight composite sandwich panels are becoming more widely used in military transport vehicles because they offer greater load bearing capabilities per unit weight and easier maintenance. In some instances, these composite sandwich panels may be subjected to high velocity impact from flying debris from a nearby explosion. While there has been much research on the low velocity impact of composite sandwich panels, very few papers deal with the issue of the high velocity impact of composite sandwich panels [1,2]. The objective of this paper is to develop an analytical model for the deformation response of a composite sandwich panel subjected to high velocity impact by a rigid blunt, cylindrical projectile. Only the mechanical constitutive equations for the facesheet and core are considered in this paper because the thermomechanical equations of state for them are presently unknown. 


\section{Problem formulation}

Consider the composite sandwich panel, as shown in Fig. 1. The facesheets are orthotropic plates of thickness $h$, and the core is crushable polymeric foam of thickness $H$. The blunt cylindrical projectile has a radius $r_{p}$, a mass $M_{o}$ and a velocity $\mathrm{V}_{\mathrm{o}}$. The projectile is assumed rigid compared to the sandwich panel. During impact, the panel experiences local indentation as well as global bending/shear deformation.

Upon impact, compressive stress waves are generated under the projectile. These stress waves must travel through the incident facesheet, core and distal facesheet before global transverse shear and bending waves can be transmitted laterally in the sandwich panel. During this phase, the problem becomes one of local indentation, i.e., the incident facesheet deflects under the projectile and the core crushes. Once the through-thickness compressive stress waves have reached the distal side of the sandwich panel, a global panel bending/shear deformation will initiate. Analytical solutions for the local and local/global transient response of the panel will be given in the following section.

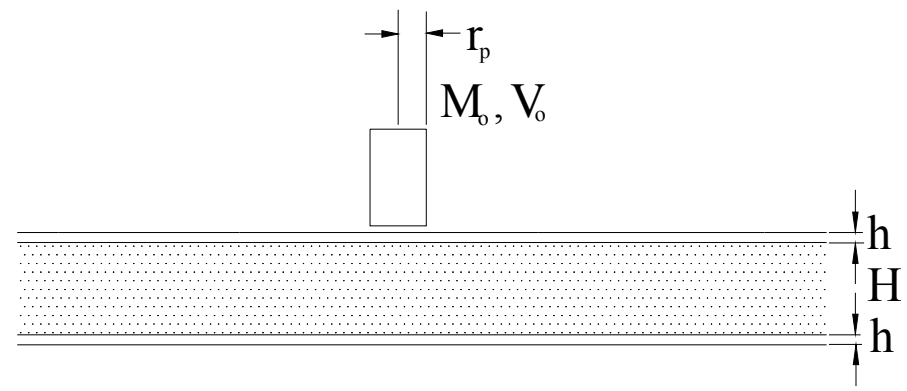

Figure 1: $\quad$ Projectile impact of the composite sandwich panel.

\section{Phase I: local indentation}

The sandwich undergoes only local indentation during Phase I. As depicted in Fig. 2, the problem is reduced to one involving projectile impact of a facesheet

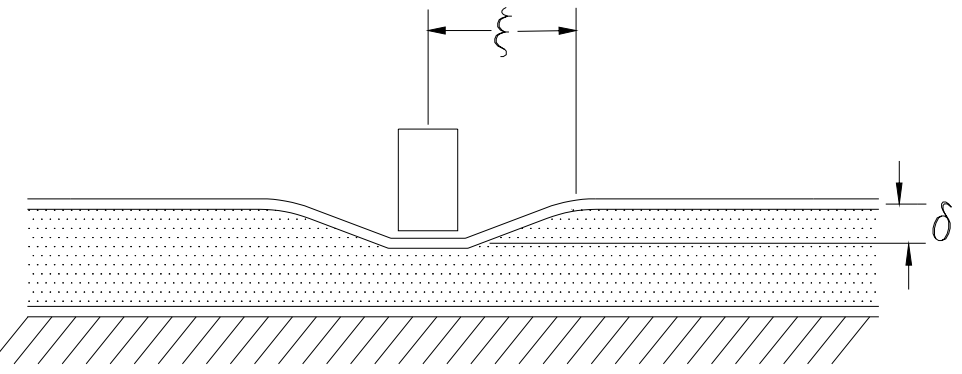

Figure 2: $\quad$ Local indentation and extent of the damage. 
resting on a foam foundation. Local indentation under the projectile is denoted $\delta$ and the lateral extent of deformation is denoted $\xi$. The duration of Phase I is determined from the wave travel time through the thickness of the panel.

\subsection{Through-thickness wave propagation}

Compressive stress waves must pass through the full thickness of the sandwich, i.e., two facesheets and core, before any response can be characterized as global bending/shear deformation. The through-thickness wave travel time is given by

$$
t_{I}=\frac{2 h}{C_{f}}+\frac{H}{C_{c}}
$$

where $C_{f}$ and $C_{c}$ are the wave speeds in the facesheet and core, respectively. The wave speed in an orthotropic plate in plane strain is given by

$$
C_{f}=\sqrt{\frac{E_{33}\left(1-v_{12}\right)}{\left[1-v_{12}-v_{32}\left(v_{13}+v_{23}\right)\right] \rho_{f}}}
$$

where $E_{i j}, v_{i j}$ and $\rho_{f}$ are the elastic modulus, Poisson's ratio and density of the orthotropic facehseet, respectively. The wave speed in the foam is determined by the amount of core crushing.

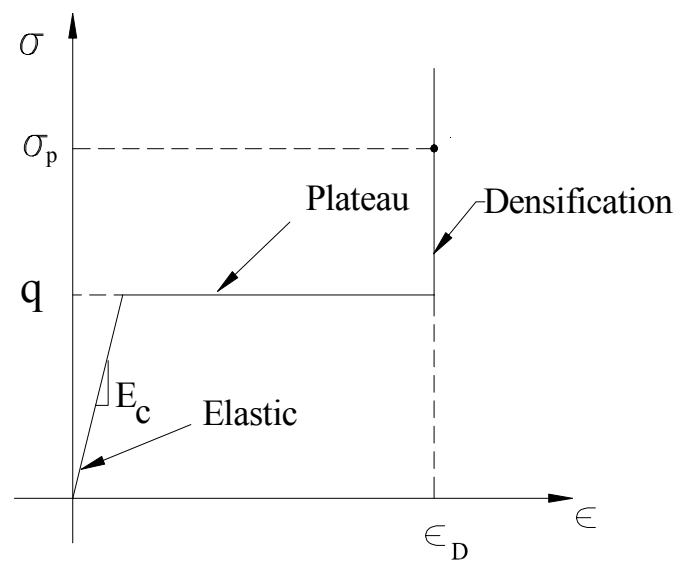

Figure 3: Compressive stress-strain curve of the polymeric foam.

A polymeric foam core is elastic-plastic with a compressive stress-strain characteristic as shown in Fig. 3 [3]. The foam is linear elastic with a compressive modulus of $E_{c}$ until yielding at a flow stress, $q$. Rapid compaction of cells causes the density to change during the plateau region until full densification has occurred at $\varepsilon_{D}$. The stress rises to a maximum plastic stress, $\sigma_{p}$, at the densification strain. Both elastic and plastic waves could therefore be generated in the foam. The elastic wave speed in the foam is given 
by $C_{e}=\sqrt{E_{c} / \rho_{c}}$ and the plastic wave speed is given by $C_{p}=\sqrt{\frac{\sigma_{p}-q}{\rho_{c} \varepsilon_{D}}}[4]$, where $\sigma_{p}$ is the stress in the densification region. The elastic wave speed is generally faster than the plastic wave speed so that $C_{c}=C_{e}$ for the throughthickness wave travel time in Eq. (1).

\subsection{Local indentation}

Local indentation is found by considering the projectile presses onto the incident facesheet resting on a rigid-plastic foundation. A single degree-of-freedom equation of motion governing the dynamics of the projectile and effective facesheet mass can be written considering the system Lagrangian. The Lagrangian $L$ for a system is defined as $L=T-\Pi$, where $T$ and $\Pi$ are the kinetic energy and potential energy of the system, respectively.

\subsubsection{Kinetic energy}

Assume the mass of the core is small compared to the mass of the facesheet. Then the kinetic energy of the system is given by

$$
T=\frac{1}{2} M_{o} V_{1}^{2}+\frac{1}{2} m_{f} V_{1}^{2}
$$

where $V_{1}$ is the velocity under the projectile at any time and $m_{f}$ is the effective mass of the facesheet. The effective mass of the facesheet is found by assuming that the projectile induces the following linear velocity field in the facesheet:

$$
\dot{w}= \begin{cases}V_{1}, & 0 \leq r \leq r_{p} \\ V_{1}\left[1-\frac{\left(r-r_{p}\right)}{\left(\xi-r_{p}\right)}\right], & r_{p} \leq r \leq \xi\end{cases}
$$

Note that $\xi$ in the above equation varies with time, i.e., the velocity field has a moving boundary and the effective facesheet mass grows as the velocity field spreads away from the impact site. Integrating the distributed kinetic energy of the facesheet and setting it equal to the equivalent kinetic energy produced by an effective facesheet mass, $m_{f}$, gives:

$$
m_{f}=\pi r_{p}^{2} \rho_{f} h+\frac{\pi \rho_{f} h}{6}\left(\xi+3 r_{p}\right)\left(\xi-r_{p}\right)
$$

\subsubsection{Potential energy}

The total potential energy of the system, $\Pi$, consists of the elastic strain energy of the facesheet, $U_{l}$, and the work dissipated in crushing the core, $D$ :

$$
\Pi=U_{l}+D
$$


Assuming in-plane deformations are negligibly small compared to transverse deflections, w, the elastic strain energy due to bending in an orthotropic facesheet is

$$
U_{l}=\frac{1}{2} \int_{S}\left[D_{11}\left(\frac{\partial^{2} w}{\partial x^{2}}\right)^{2}+2 D_{12} \frac{\partial^{2} w}{\partial x^{2}} \frac{\partial^{2} w}{\partial y^{2}}+D_{22}\left(\frac{\partial^{2} w}{\partial y^{2}}\right)^{2}+4 D_{66}\left(\frac{\partial^{2} w}{\partial x \partial y}\right)^{2}\right] d S
$$

where $D_{i j}$ is the bending stiffness of the facesheet and $\mathrm{S}$ is the area associated with indentation.

The work dissipated in foam core crushing is given by

$$
D=\int_{S} q w d S
$$

where $q$ is the constant, compressive flow stress in the foam.

Finite element analysis has shown that the transverse deflection of the facesheet may be assumed as follows:

$$
w(r)= \begin{cases}\delta, & 0 \leq r \leq r_{p} \\ \delta\left[1-\frac{\left(\mathrm{r}-\mathrm{r}_{\mathrm{p}}\right)}{\left(\xi-r_{p}\right)}\right]^{2}, & r_{p} \leq r \leq \xi\end{cases}
$$

where $r^{2}=x^{2}+y^{2}$. Transforming derivatives from rectangular to polar coordinates, allows one to calculate the strain energy due to facesheet bending as

$$
U_{l}=\frac{C_{1} \pi \delta^{2}}{4} \frac{\left(\xi+r_{p}\right)}{\left(\xi-r_{p}\right)^{3}}-\frac{C_{2} \pi \delta^{2}}{2\left(\xi-r_{p}\right)^{2}}+\frac{C_{1} \pi \delta^{2}}{4\left(\xi-r_{p}\right)^{4}}\left[-3 \xi^{2}+4 \xi r_{p}-r_{p}^{2}-2 \xi^{2} \ln \left(\frac{\xi}{r_{p}}\right)\right]
$$

where $C_{1}=3\left(D_{11}+D_{22}\right)+2 D_{12}+4 D_{66}$ and $C_{2}=D_{11}+D_{22}+6 D_{12}-4 D_{66}$.

The work dissipated in foam core crushing is also given by

$$
D=2 \pi q \delta\left[\frac{\left(\xi^{2}-r_{p}^{2}\right)}{2}+\frac{\left(\xi^{4}-r_{p}^{4}\right)}{4 \xi^{2}}-\frac{2\left(\xi^{3}-r_{p}^{3}\right)}{3 \xi}\right]
$$

\subsubsection{Equation of motion}

Lagrange's equation of motion for the projectile and effective facesheet mass is

$$
\frac{\partial}{\partial t}\left(\frac{\partial L}{\partial V_{1}}\right)-\frac{\partial L}{\partial \delta}=\left(M_{o}+m_{f}\right) \frac{d V_{1}}{d t}+V_{1} \frac{d m_{f}}{d t}+\frac{\partial \Pi}{\partial \delta}=0
$$

where $V_{1}=\frac{d \delta}{d t}$. Substituting $m_{f}$ and $\Pi$ into Eq. (12) gives

$$
\begin{aligned}
& \left(M_{o}+m_{f}\right) \frac{d^{2} \delta}{d t^{2}}+\frac{\pi \rho_{f} h}{3 t} \xi\left(\xi+r_{p}\right) \frac{d \delta}{d t}+\frac{C_{1} \pi \delta}{2} \frac{\left(\xi+r_{p}\right)}{\left(\xi-r_{p}\right)^{3}}-\frac{C_{2} \pi \delta}{\left(\xi-r_{p}\right)^{2}} \\
& +\frac{C_{1} \pi \delta}{2\left(\xi-r_{p}\right)^{4}}\left[-3 \xi^{2}+4 \xi r_{p}-r_{p}^{2}-2 \xi^{2} \ln \left(\frac{\xi}{r_{p}}\right)\right]+2 \pi q\left[\frac{\left(\xi^{2}-r_{p}^{2}\right)}{2}+\frac{\left(\xi^{4}-r_{p}^{4}\right)}{4 \xi^{2}}-\frac{2\left(\xi^{3}-r_{p}^{3}\right)}{3 \xi}\right]=0
\end{aligned}
$$


where it is assumed that $\dot{\xi}=\xi / t$. Conservation of linear momentum relates the projectile and effective facesheet mass velocity, $V_{1}$, with $\xi$ :

$$
M_{o} V_{o}=M_{o} V_{1}+\left[\pi r_{p}^{2} \rho_{f} h+\frac{\pi \rho_{f} h}{3}\left(\xi+2 r_{p}\right)\left(\xi-r_{p}\right)\right] V_{1}
$$

Equation (13) becomes a nonlinear second order differential equation in $\delta$ when Eq. (14) is used to eliminate $\xi$. The initial conditions for Eq. (13) are $\delta(0)=0$ and $V_{1}(0)=\dot{\delta}(0)=\frac{M_{o} V_{o}}{\left(M_{o}+\pi r_{p}^{2} \rho_{f} h\right)}$.

\section{Phase I: global bending/shear}

At the end of Phase I, transverse shear waves propagate from the point of impact across the sandwich panel. The global panel deflection is denoted $\Delta$ and the lateral extent of global deformation is denoted $\Xi$ as shown in Fig. 4.

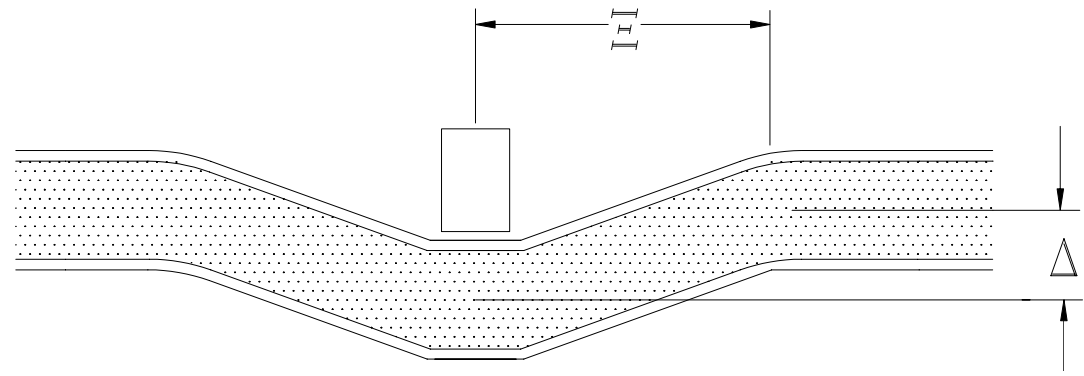

Figure 4: Global deformation and extent of the damage.

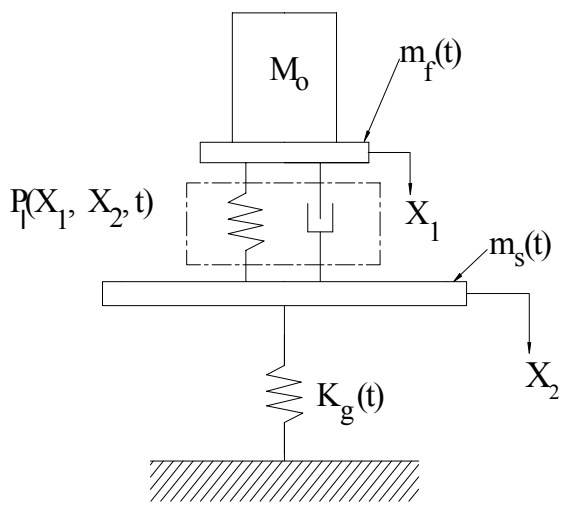

Figure 5: $\quad$ Coupled local and global deformation model. 
The two degree-of-freedom model shown in Fig. 5 is considered for combined local indentation and global panel bending/shear deformations. Degrees of freedom, $X_{1}$ and $X_{2}$, are related to the position of the projectile and incident facesheet, and the deflection of the sandwich panel under the projectile if there were no local indentation or core crushing, respectively. Therefore, $\delta=X_{1}-X_{2}$ and $\Delta=X_{2}$. Applying Lagrange's equations of motion results in two equations of motion:

$$
\begin{aligned}
& \frac{\partial}{\partial t}\left(\frac{\partial L}{\partial V_{1}}\right)-\frac{\partial L}{\partial X_{1}}=0 \\
& \frac{\partial}{\partial t}\left(\frac{\partial L}{\partial V_{2}}\right)-\frac{\partial L}{\partial X_{2}}=0
\end{aligned}
$$

where $V_{2}=\frac{d X_{2}}{d t}$.

\subsection{Kinetic energy}

Assume the mass of the core is small compared to the mass of the facesheet and neglect rotary inertia of the sandwich panel. Then the kinetic energy of the system is given by

$$
T=\frac{1}{2} M_{o} V_{1}^{2}+\frac{1}{2} m_{f} V_{1}^{2}+\frac{1}{2} m_{s} V_{2}^{2}
$$

where $m_{s}$ is the effective mass of the sandwich and $V_{2}$ is the velocity of the sandwich under the projectile. The effective mass of the sandwich is calculated by again assuming a linear velocity field for the sandwich:

$$
\dot{w}=V_{2}\left(1-\frac{r}{\Xi}\right)
$$

From the above velocity field, one finds that the effective sandwich mass is

$$
m_{s}=\frac{\pi}{6}\left(\rho_{f} h+\rho_{c} H\right) \Xi^{2}
$$

Note that only one facesheet mass is in Eq. (19) because an effective mass of the incident facesheet has already been considered.

\subsection{Global bending/shear energy}

The elastic strain energy due to bending and shear of a symmetric sandwich panel with orthotropic facesheet is

$$
\begin{aligned}
U_{g}=\int_{S} & \left\{\frac{D_{11}^{s}}{2}\left(\frac{\partial \bar{\alpha}}{\partial x}\right)^{2}+D_{12}^{s}\left(\frac{\partial \bar{\beta}}{\partial y}\right)\left(\frac{\partial \bar{\alpha}}{\partial x}\right)+\frac{D_{22}^{s}}{2}\left(\frac{\partial \bar{\beta}}{\partial y}\right)^{2}+A_{55}^{s}\left[\frac{\bar{\alpha}^{2}}{2}+\bar{\alpha} \frac{\partial w}{\partial x}+\frac{1}{2}\left(\frac{\partial w}{\partial x}\right)^{2}\right]\right. \\
& \left.+A_{44}^{s}\left[\frac{\bar{\beta}^{2}}{2}+\bar{\beta} \frac{\partial w}{\partial y}+\frac{1}{2}\left(\frac{\partial w}{\partial y}\right)^{2}\right]+D_{66}^{s}\left[\frac{1}{2}\left(\frac{\partial \bar{\alpha}}{\partial y}\right)^{2}+\frac{\partial \bar{\alpha}}{\partial y} \frac{\partial \bar{\beta}}{\partial x}+\frac{1}{2}\left(\frac{\partial \bar{\beta}}{\partial x}\right)^{2}\right]\right\} d S
\end{aligned}
$$


where $\mathrm{w}$ is again used to express transverse deflections, $\bar{\alpha}$ and $\bar{\beta}$ are shear angles associated with the x- and y-directions, respectively, $D_{i j}^{s}$ is the sandwich bending stiffness matrix, and $A_{44}^{s}$ and $A_{55}^{s}$ are the transverse shear stiffnesses. The superscript " $\mathrm{s}$ " is used to denote the sandwich.

Assume the following global transverse deflection and shear rotations:

$$
w(r)= \begin{cases}\Delta\left(1-\left(\frac{r}{\Xi}\right)^{2}\right)^{2}, & 0 \leq r \leq \Xi \\ 0, & r \geq \Xi\end{cases}
$$

and

$$
\bar{\alpha}(r)=\bar{\beta}(r)= \begin{cases}\frac{4 \alpha_{\mathrm{o}} r}{\Xi^{2}}(\Xi-r), & 0 \leq r \leq \Xi \\ 0, & r \geq \Xi\end{cases}
$$

where $\Delta$ is the global deflection under the projectile and $\alpha_{o}$ is the shear rotation at the center of the panel.

Substituting derivatives of the expressions in Eqs. (21) and (22) into

Eq. (20) gives the following expression for the strain energy:

$$
\begin{aligned}
U_{g}=\frac{4}{3}\left[\pi\left(D_{11}^{s}+D_{22}^{s}\right)+4 D_{12}^{s}+2(\pi+2) D_{66}^{s}\right] \alpha_{o}^{2}+ \\
\frac{1}{105}\left(A_{44}^{s}+A_{55}^{s}\right)\left(-176 \Xi \alpha_{o} \Delta+28 \pi \Xi^{2} \alpha_{o}^{2}+35 \pi \Xi \Delta^{2}\right)
\end{aligned}
$$

\subsection{Equations of motion}

Satisfying Lagrange's equations stated in Eqs. (15) and (16) one gets

$$
\begin{aligned}
& \left(M_{o}+m_{f}\right) \frac{d^{2} X_{1}}{d t^{2}}+\frac{\pi \rho_{f} h}{3 t} \xi\left(\xi+r_{p}\right) \frac{d X_{1}}{d t}+\frac{C_{1} \pi\left(X_{1}-X_{2}\right)}{2} \frac{\left(\xi+r_{p}\right)}{\left(\xi-r_{p}\right)^{3}}-\frac{C_{2} \pi\left(X_{1}-X_{2}\right)}{\left(\xi-r_{p}\right)^{2}} \\
& +\frac{C_{1} \pi\left(X_{1}-X_{2}\right)}{2\left(\xi-r_{p}\right)^{4}}\left[-3 \xi^{2}+4 \xi r_{p}-r_{p}^{2}-2 \xi^{2} \ln \left(\frac{\xi}{r_{p}}\right)\right]+2 \pi q\left[\frac{\left(\xi^{2}-r_{p}^{2}\right)}{2}+\frac{\left(\xi^{4}-r_{p}^{4}\right)}{4 \xi^{2}}-\frac{2\left(\xi^{3}-r_{p}^{3}\right)}{3 \xi}\right]=0
\end{aligned}
$$

and

$$
\begin{aligned}
& m_{s} \frac{d^{2} X_{2}}{d t^{2}}+\frac{\pi\left(\rho_{f} h+\rho_{c} H\right)}{3 t} \Xi^{2} \frac{d X_{2}}{d t}-\frac{C_{1} \pi\left(X_{1}-X_{2}\right)}{2} \frac{\left(\xi+r_{p}\right)}{\left(\xi-r_{p}\right)^{3}}+\frac{C_{2} \pi\left(X_{1}-X_{2}\right)}{\left(\xi-r_{p}\right)^{2}} \\
& -\frac{C_{1} \pi\left(X_{1}-X_{2}\right)}{2\left(\xi-r_{p}\right)^{4}}\left[-3 \xi^{2}+4 \xi r_{p}-r_{p}^{2}-2 \xi^{2} \ln \left(\frac{\xi}{r_{p}}\right)\right] \\
& -2 \pi q\left[\frac{\left(\xi^{2}-r_{p}^{2}\right)}{2}+\frac{\left(\xi^{4}-r_{p}^{4}\right)}{4 \xi^{2}}-\frac{2\left(\xi^{3}-r_{p}^{3}\right)}{3 \xi}\right]+\frac{1}{105}\left(A_{44}^{s}+A_{55}^{s}\right)\left(-176 \Xi \alpha_{o}+70 \pi \Xi X_{2}\right)=0
\end{aligned}
$$


where it is assumed that $\dot{\Xi}=\Xi / t$. Requiring that $\frac{\partial \Pi}{\partial \alpha_{o}}=0$ gives

$$
\alpha_{o}=\frac{22\left(A_{44}^{s}+A_{55}^{s}\right) \Xi X_{2}}{35\left[\pi\left(D_{11}^{s}+D_{22}^{s}\right)+4 D_{12}^{s}+2(\pi+2) D_{66}^{s}\right]+7 \pi\left(A_{44}^{s}+A_{55}^{s}\right) \Xi^{2}}
$$

Conservation of linear momentum also gives

$$
M_{o} V_{o}=M_{o} V_{1}+\left[\pi r_{p}^{2} \rho_{f} h+\frac{\pi \rho_{f} h}{3}\left(\xi+2 r_{p}\right)\left(\xi-r_{p}\right)\right] V_{1}+\frac{\pi\left(\rho_{f} h+\rho_{c} H\right)}{3} \Xi^{2} V_{2}
$$

Finally, the kinetic energy of the sandwich with both facesheets is compared to the sum of the kinetic energy of the facesheet and the sandwich with only the distal facesheet:

$$
\begin{aligned}
\frac{1}{2}\left[\frac{\pi\left(2 \rho_{f} h+\rho_{c} H\right)}{6} \Xi^{2}\right] V_{1}^{2}=\frac{1}{2}\left[\frac{\pi\left(\rho_{f} h+\rho_{c} H\right)}{6} \Xi^{2}\right] V_{2}^{2} \\
+\frac{1}{2}\left[\pi r_{p}^{2} \rho_{f} h+\frac{\pi \rho_{f} h}{6}\left(\xi+3 r_{p}\right)\left(\xi-r_{p}\right)\right] V_{1}^{2}
\end{aligned}
$$

Equations (24) - (28) are solved with the following initial conditions: $X_{1}\left(t_{I}\right)=X_{10}, \dot{X}_{1}\left(t_{I}\right)=\dot{X}_{10}, X_{2}\left(t_{I}\right)=0$, and $\dot{X}_{2}\left(t_{I}\right)=0$, where $t_{I}$ is defined in Eq. (1) and $X_{10}$ and $\dot{X}_{10}$ are taken at the end of Phase I.

\section{An example}

As an example, consider a fully clamped sandwich panel made of E-glass vinyl ester facesheets and PVC H100 foam core, with a panel radius of $250 \mathrm{~mm}$, facesheet thickness of $2 \mathrm{~mm}$, and core thickness of $25 \mathrm{~mm}$. Material properties for the E-glass vinyl ester and the PVC H100 foam are given in Table 1.

Let the sandwich panel undergo impact by a rigid cylindrical rod of radius 2.5 $\mathrm{mm}$, mass $0.5 \mathrm{~kg}$, and velocity $40 \mathrm{~m} / \mathrm{s}$. This impact problem was modeled using ABAQUS Explicit using continuum C3D8R elements for both the facesheets and the foam. The PVC H100 foam was modeled as crushable foam with volumetric hardening. Additional foam properties, such as the plastic hardening curve were taken from [5].

The analytical solution for the incident facesheet under the projectile and global panel deflections is compared to FEA results in Fig. 6. The deflection of the distal facesheet under the projectile was taken as the global panel deflection in FEA. The analytical deflections are about $15 \%$ lower than FEA predictions. 
Table 1: $\quad$ Facesheet and foam material properties.

\begin{tabular}{|l|l|l|}
\hline & E-Glass/Vinyl Ester & Divinycell H100 \\
\hline Density $\left(\mathrm{kg} / \mathrm{m}^{3}\right)$ & 1391.3 & 100 \\
\hline Thickness $(\mathrm{mm})$ & 2 & 25 \\
\hline $\mathrm{E}_{11}(+)(\mathrm{GPa})$ & 17 & 0.126 \\
\hline $\mathrm{E}_{22}(+)(\mathrm{GPa})$ & 17 & 0.126 \\
\hline $\mathrm{E}_{33}(+)(\mathrm{GPa})$ & 8.5 & 0.126 \\
\hline $\mathrm{E}_{11}(-)(\mathrm{GPa})$ & 17 & 0.035 \\
\hline $\mathrm{E}_{22}(-)(\mathrm{GPa})$ & 17 & 0.035 \\
\hline $\mathrm{E}_{33}(-)(\mathrm{GPa})$ & 8.5 & 0.035 \\
\hline$v_{12}$ & 0.13 & 0 \\
\hline$v_{13}$ & 0.28 & 0 \\
\hline$v_{23}$ & 0.28 & 0 \\
\hline $\mathrm{G}_{12}=\mathrm{G}_{21}(\mathrm{GPa})$ & 4.0 & 0.0175 \\
\hline $\mathrm{G}_{23}=\mathrm{G}_{32}(\mathrm{GPa})$ & 4.2 & 0.0175 \\
\hline $\mathrm{G}_{13}=\mathrm{G}_{31}(\mathrm{GPa})$ & 4.2 & 0.0175 \\
\hline $\mathrm{q}(\mathrm{MPa})$ & -- & 1.66 \\
\hline$\varepsilon_{\mathrm{D}}$ & -- & 0.8 \\
\hline
\end{tabular}

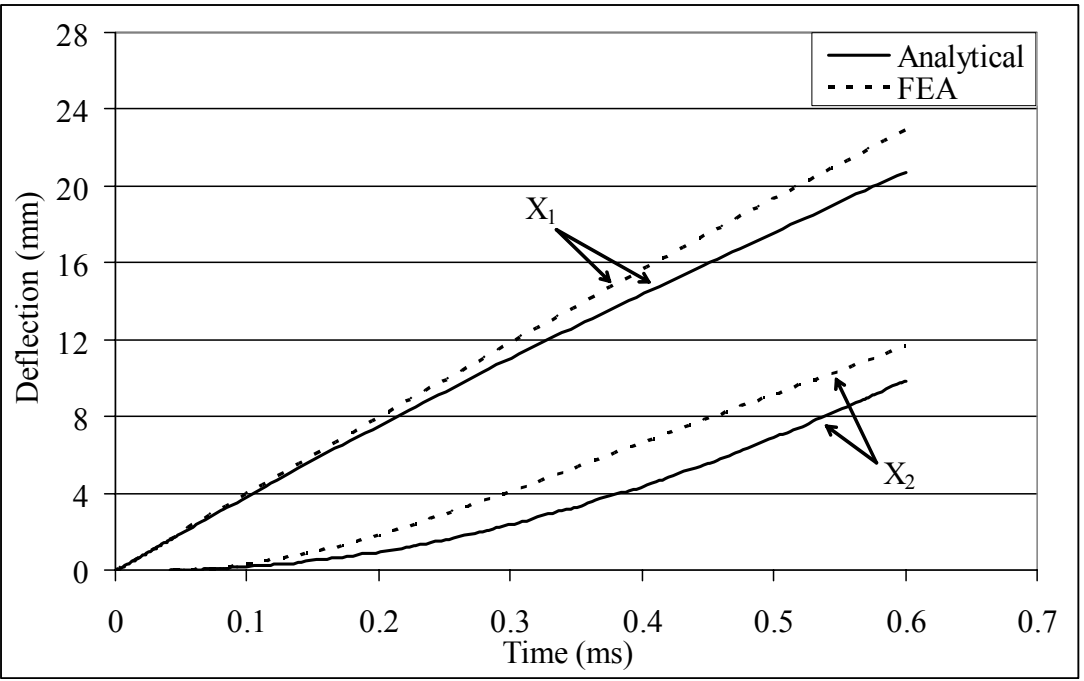

Figure 6: Transient deflections of incident and distal facesheets under the projectile.

\section{Conclusions}

A two degree-of-freedom model was developed and used to obtain the local indentation and global deformation response of a sandwich panel made with Eglass vinyl ester facesheets and PVC H100 foam core and subjected to high 
velocity impact by a rigid blunt, cylindrical projectile. The analytical solution for the local indentation and global deflection under the projectile was found to be $15 \%$ lower than FEA results.

\section{Acknowledgement}

This work was supported by Dr. Yapa Rajapakse at the Office of Naval Research under grant N00014-07-1-0423.

\section{References}

[1] Skvortsov, V., Kepler, J. \& Bozhevolnaya, E. Energy Partition for Ballistic Penetration of Sandwich Panels, Int. J. Impact Engng., Vol. 28, pp. 697716, 2003.

[2] Tagarielli, V.L., Deshpande, V.S. \& Fleck, N.A. The dynamic response of composite sandwich beams to transverse impact, Int. J. Solids Struct., Vol. 44, pp. 2442-2457, 2007.

[3] Gibson, L.J. \& Ashby, M.F. Cellular Solids Structures and Properties, Cambridge University Press, Cambridge, 1999.

[4] Ashby, M.F., Evans, A.G., Fleck, N.A., Gibson, L.J., Hutchinson, J.W. \& Wadley, H.N.G. Metal Foams: A Design Guide, Butterworth Heinemann, London, 2000.

[5] Mines, R.A.W. \& Alias, A. Numerical simulation of the progressive collapse of polymer composite sandwich beams under static loading, Composites Part A, Vol. 33, pp. 11-26, 2002. 